\title{
The value of multiple tests of respiratory muscle strength
}

\author{
Joerg Steier, Sunny Kaul, John Seymour, Caroline Jolley, Gerrard Rafferty, William Man, Yuan \\ M Luo, Michael Roughton, Michael I Polkey, John Moxham
}

Thorax 2007;62:975-980. doi: 10.1136/thx.2006.072884

See end of article for authors' affiliations

Correspondence to: Dr Joerg Steier, Respiratory Muscle Laboratory, King's College London School of Medicine, King's College Hospital, Denmark Hill, London SE5 9PJ, UK; joerg. steier@kcl.ac.uk

Received 5 October 2006 Accepted 10 April 2007 Published Online First 8 June 2007

\begin{abstract}
Background: Respiratory muscle weakness is an important clinical problem. Tests of varying complexity and invasiveness are available to assess respiratory muscle strength. The relative precision of different tests in the detection of weakness is less clear, as is the value of multiple tests.

Methods: The respiratory muscle function tests of clinical referrals who had multiple tests assessed in our laboratories over a 6-year period were analysed. Thresholds for weakness for each test were determined from published and in-house laboratory data. The patients were divided into three groups: those who had all relevant measurements of global inspiratory muscle strength (group $A, n=182$ ), those with full assessment of diaphragm strength (group $B, n=264$ ) and those for whom expiratory muscle strength was fully evaluated (group $C, n=60$ ). The diagnostic outcome of each inspiratory, diaphragm and expiratory muscle test, both singly and in combination, was studied and the impact of using more than one test to detect weakness was calculated.

Results: The clinical referrals were primarily for the evaluation of neuromuscular diseases and dyspnoea of unknown cause. A low maximal inspiratory mouth pressure (PImax) was recorded in $40.1 \%$ of referrals in group A, while a low sniff nasal pressure (Sniff Pnasal) was recorded in $41.8 \%$ and a low sniff oesophageal pressure (Sniff Poes) in 37.9\%. When assessing inspiratory strength with the combination of all three tests, $29.6 \%$ of patients had weakness. Using the two non-invasive tests (PImax and Sniff Pnasal) in combination, a similar result was obtained (low in 32.4\%). Combining Sniff Pdi (low in 68.2\%) and Twitch Pdi (low in 67.4\%) reduced the diagnoses of patients with diaphragm weakness to $55.3 \%$ in group B. $38.3 \%$ of the patients in group $C$ had expiratory muscle weakness as measured by maximum expiratory pressure (PEmax) compared with $36.7 \%$ when weakness was diagnosed by cough gastric pressure (Pgas), and $28.3 \%$ when assessed by Twitch T10. Combining all three expiratory muscle tests reduced the number of patients diagnosed as having expiratory muscle weakness to $16.7 \%$.

Conclusion: The use of single tests such as PImax, PEmax and other available individual tests of inspiratory, diaphragm and expiratory muscle strength tends to overdiagnose weakness. Combinations of tests increase diagnostic precision and, in the population studied, they reduced the diagnosis of inspiratory, specific diaphragm and expiratory muscle weakness by 19-56\%. Measuring both PImax and Sniff Pnasal resulted in a relative reduction of $19.2 \%$ of patients falsely diagnosed with inspiratory muscle weakness. The addition of Twitch Pdi to Sniff Pdi increased diagnostic precision by a smaller amount (18.9\%). Having multiple tests of respiratory muscle function available both increases diagnostic precision and makes assessment possible in a range of clinical circumstances.
\end{abstract}

M easurement of respiratory muscle strength is clinically useful in the assessment of selected patients, most commonly those with neuromuscular diseases or unexplained breathlessness. ${ }^{12}$

Maximum inspiratory (PImax) and expiratory (Pemax) pressures are most frequently measured. Pimax and Pemax are simple quick tests, and high values exclude clinically significant weakness. However, low values are common and may reflect poor technique or effort rather than muscle weakness. ${ }^{3}$

Additional tests are available which are likely to improve diagnostic precision but are more complex and invasive. ${ }^{4-8}$ We have reviewed our test results in patients referred for assessment of respiratory muscle strength to determine the value of multiple respiratory muscle tests. We hypothesised that multiple tests might reduce the number of patients erroneously diagnosed as having muscle weakness.

\section{METHODS}

Test results of clinical referrals made to the respiratory muscle laboratories of King's College and Brompton Hospitals between 2000 and 2006 were analysed. Tests were undertaken according to established methods as described in the ATS/ERS joint statement. ${ }^{3}$ The following tests were used.

\section{Maximum inspiratory pressure (PImax)}

Maximum inspiratory pressures were measured from functional residual capacity in the standard way ${ }^{3}$ with the patient seated, wearing a nose-clip and using a flanged mouthpiece (P K Morgan Ltd, Rainham, UK). Repeated efforts were made until consistent results were achieved and the numerically largest pressure noted. The average of the pressure was measured over 1 s. $^{3}$

Several publications report normal values using a flanged mouthpiece. ${ }^{9-12}$ Weakness was defined as the mean normal value minus 1.96 standard deviations based on the study by Wilson et al (table 1). ${ }^{9}$ This number reflects the $100 \%$ line in the figures in the Results section.

\section{Sniff manoeuvres}

Balloon catheters for the measurement of pressure (Cooper Surgical, Connecticut, USA) lubricated with $2 \%$ lidocaine gel were introduced via one nostril into the oesophagus and stomach as described by Baydur et al. ${ }^{13}$ The distal balloon (filled

Abbreviations: Pdi, transdiaphragmatic pressure; PEmax, maximum expiratory pressure; Pgas, gastric pressure; PImax, maximum inspiratory pressure; Pnasal, nasal pressure; Poes, oesophageal pressure 


\begin{tabular}{|c|c|c|c|c|}
\hline Test & Sex & Calculation & $\begin{array}{l}\text { Cut off } \\
\left(\mathrm{cm} \mathrm{H}_{2} \mathrm{O}\right)\end{array}$ & $\begin{array}{l}\text { Rounded } \\
\left(\mathrm{cm} \mathrm{H}_{2} \mathrm{O}\right)\end{array}$ \\
\hline \multirow[t]{2}{*}{ PImax } & $M$ & $10.4-1.96 \times 3.0 \mathrm{kPa}$ & 44.8 & 45 \\
\hline & $\mathrm{F}$ & $7.2-1.96 \times 2.1 \mathrm{kPa}$ & 31.6 & 30 \\
\hline \multirow[t]{2}{*}{ Sniff Poes } & M & $105-1.96 \times 26 \mathrm{~cm} \mathrm{H}_{2} \mathrm{O}$ & 54.0 & 55 \\
\hline & $\mathrm{F}$ & $92-1.96 \times 22 \mathrm{~cm} \mathrm{H}_{2} \mathrm{O}$ & 48.9 & 50 \\
\hline \multirow[t]{2}{*}{ Sniff Pnasal } & M & $0.91 \times 55 \mathrm{~cm} \mathrm{H}_{2} \mathrm{O}$ & 50.1 & 50 \\
\hline & $\mathrm{F}$ & $0.91 \times 50 \mathrm{~cm} \mathrm{H}_{2} \mathrm{O}$ & 45.5 & 45 \\
\hline \multirow[t]{2}{*}{ Sniff Pdi } & M & $148-1.96 \times 24 \mathrm{~cm} \mathrm{H}_{2} \mathrm{O}$ & 101.0 & 100 \\
\hline & $\mathrm{F}$ & $121-1.96 \times 25 \mathrm{~cm} \mathrm{H}_{2} \mathrm{O}$ & 72.0 & 70 \\
\hline Twitch Pdi & $M \& F$ & $28-1.96 \times 5 \mathrm{~cm} \mathrm{H}_{2} \mathrm{O}$ & 18.2 & 18 \\
\hline \multirow{2}{*}{ Pemax } & M & $14.4-1.96 \times 3.3 \mathrm{kPa}$ & 80.5 & 80 \\
\hline & $\mathrm{F}$ & $9.1-1.96 \times 1.6 \mathrm{kPa}$ & 61.1 & 60 \\
\hline \multirow{2}{*}{ Cough Pgas } & M & $214.4-1.96 \times 42.2 \mathrm{~cm} \mathrm{H}_{2} \mathrm{O}$ & 0131.7 & 130 \\
\hline & $\mathrm{F}$ & $165.1-1.96 \times 34.8 \mathrm{~cm} \mathrm{H}_{2} \mathrm{O}$ & O 96.9 & 95 \\
\hline Twitch T10 & $M \& F$ & $\begin{array}{l}x=1.6-1.96 \times 0.20 \\
\text { re-transformation: } y=10^{x}\end{array}$ & 16.1 & 16 \\
\hline
\end{tabular}

PImax, maximum inspiratory pressure; Poes, oesophageal pressure; Pnasal, nasal pressure; Pdi, transdiaphragmatic pressure, PEmax, maximum expiratory pressure; Pgas, gastric pressure.

with $2 \mathrm{ml}$ air) measured the gastric pressure (Pgas) and the proximal balloon (filled with $0.5 \mathrm{ml}$ air) measured the oesophageal pressure (Poes). Transdiaphragmatic pressure (Pdi) was derived by calculating the difference between Poes and Pgas. Differential pressure transducers were connected to amplifiers (Validyne, Northridge, California, USA) that transmitted the signal to a computer (Apple iMac Computers, Cupertino, California, USA). LabVIEW4.1 was used for recording and analysis of data (National Instruments, Austin, Texas, USA). Later referrals were analysed using 16-Channel Powerlab with CHART V software (ADInstruments, Colorado Springs, Colorado, USA).

\section{Sniff oesophageal pressure (Sniff Poes)}

Sniff manoeuvres were performed with the patient seated and the balloon catheters in place as described above. ${ }^{3}$ At least 5-10 maximal sniffs were measured and the largest numerical pressure was noted. Data of Laroche et $a l^{5}$ were used to calculate the normal cut off values (table 1).

\section{Sniff nasal pressure (Sniff Pnasal)}

A plug, used to obstruct one nostril, incorporated the distal 2$3 \mathrm{~cm}$ of a $30 \mathrm{~cm}$ polyethylene catheter with a $2 \mathrm{~mm}$ internal diameter (Intersurgical Scientific Instruments, Oxford, UK). The proximal end of the catheter was attached to a pressure transducer (Validyne). At least 5-10 maximal sniffs were performed until a consistent value of sniff pressure was reached; the highest numerical pressure was taken. Heritier et al ${ }^{4}$ described a close relationship between Sniff Pnasal and Sniff Poes $(r=0.99)$ in normal subjects without nasal obstruction. The ratio of Sniff Pnasal to Sniff Poes was 0.91. The lower limit of normal cut off values were derived using the values from the Sniff Poes test ${ }^{5}$ multiplied by 0.91 , the ratio of Sniff Pnasal/Sniff Poes (table 1$){ }^{4}$

\section{Sniff transdiaphragmatic pressure (Sniff Pdi)}

Pressure catheters were placed and maximal sniff manoeuvres performed as described above. The highest numerical pressure of 5-10 consistent sniffs was taken. Normal cut off values refer to the data of Miller et $a l^{8}$ who described normal values for the sniff Pdi test (table 1).

\section{Twitch transdiaphragmatic pressure (Twitch Pdi)}

Twitch transdiaphragmatic pressure was measured following magnetic stimulation of the phrenic nerves via a bilateral anterolateral approach at functional residual capacity. ${ }^{6}{ }^{14} 15$ The patient was seated, wearing a noseclip, and the mouth was closed. For magnetic stimulation a Magstim 200 (Magstim Co Ltd, Whitland, UK) with a $43 \mathrm{~mm}$ double coil (P/N9784-00; Magstim Co Ltd) was used. After achieving a supramaximal stimulus, at least five consistent twitches were recorded and the average Twitch Pdi calculated.

Luo et al investigated twitch Pdi in normal subjects and found it to be $28(5) \mathrm{cm} \mathrm{H}_{2} \mathrm{O}$ (table 1). ${ }^{6}$ No distinction was made for normal values between the sexes because the available literature on sex differences is insufficient.

\section{Maximum expiratory pressure (PEmax)}

Maximum expiratory pressures were measured from total lung capacity in the standard way with the patient seated, wearing a noseclip and using a flanged mouthpiece (P K Morgan Ltd). ${ }^{3} 9$ Repeated efforts were made until consistent results were achieved and the numerically largest pressure averaged over l s was measured. ${ }^{3}$ Several studies have reported normal values using a flanged mouthpiece. ${ }^{9-12}$ Normal cut off values refer to the study of Wilson et al (table 1$){ }^{9}$

\section{Cough gastric pressure (Cough Pgas)}

Pressure balloons were positioned as described above for sniff manoeuvres. The cough manoeuvre was performed as previously reported, breathing in deeply first, with the patient seated and wearing a noseclip. ${ }^{3}$ Coughs were repeated at least 5-10 times until consistent measurements were achieved. The numerically highest value was taken, measuring from relaxed end-expiratory baseline gastric pressure to peak pressure during the cough. Man et al described cough gastric pressures in 99 healthy volunteers, enabling normal cut off values to be calculated (table 1).

\section{Twitch T10 gastric pressure (Twitch T10)}

Gastric pressure was measured as described for sniff manoeuvres and magnetic stimulation of the thoracic nerve roots was performed with a $90 \mathrm{~mm}$ circular coil (P/N9784-00; Magstim Co Ltd) placed with its centre over the 10th thoracic vertebra in the mid line. ${ }^{16}$ The manoeuvre was undertaken at functional residual capacity with the patient seated, wearing a noseclip and the mouth closed. Twitches were repeated at least 5-10 times until consistent measurements were obtained and an average Twitch T10 was calculated. There are few normal data reported for this test. Our laboratory data are from 65 normal subjects (41 men and 24 women) of mean (SD) age 51 (16) years and body mass index 25.6 (3.6) $\mathrm{kg} / \mathrm{m}^{2}$. The results are not normally distributed but are positively skewed. The median was $39.4 \mathrm{~cm} \mathrm{H}_{2} \mathrm{O}$ (interqartile range $26.6 \mathrm{~cm} \mathrm{H}_{2} \mathrm{O}$ ). The cut off value for weakness was calculated after transformation of the data into a log-normal distribution (mean $1.6(0.20)$ ). The mean minus 1.96 standard deviations was calculated and the parameter retransformed $\left(y=10^{x}\right)$ to give the cut off value in table 1. As for Twitch Pdi, no distinction was made between sexes because of the relatively limited data.

The outcome of the respiratory muscle tests in diagnosing weakness was studied singly and in combination. Crosstabulation identified the diagnosis of weakness for each test and the added value of using more than one test in detecting respiratory muscle weakness was determined.

\section{Analysis of data}

For the purposes of analysis, patient data were used for comparison only if all of the global inspiratory, specific diaphragm or expiratory muscle tests were performed. For inspiratory muscle tests (Pimax, Sniff Pnasal and Sniff Poes) this was 182 of the referrals (group A), for diaphragm specific 
Table 2 Diagnoses of all patients

\begin{tabular}{lr}
\hline Neuromuscular disease & 156 \\
Dyspnoea of unknown origin & 94 \\
Chronic obstructive pulmonary diseases (COPD) & 45 \\
Rheumatological disease & 37 \\
Chest wall disease (acquired and congenital) & 22 \\
Other restrictive lung diseases & 15 \\
Obesity hypoventilation & 14 \\
Malignancy & 7 \\
Others & 23 \\
\hline
\end{tabular}

tests (Sniff Pdi and Twitch Pdi) 264 (Group B), and for expiratory muscle tests (Pemax, Cough Pgas and Twitch T10) 60 (Group C). Individual test results were judged relative to the diagnosis achieved by combining all relevant tests.

For statistical analysis and graph plots, SPSS Version 13.0 (SPSS Inc, Chicago, Illinois, USA) was used. The results are given as mean (SD) for all tests except Twitch T10 values for which the results are given as median (interquartile range, IQR) because of non-normal distribution of the data. Correlation coefficients were calculated for all tests (Pearson's correlation coefficient), except Twitch T10 for which Spearman's correlation coefficient was used.

Values for single tests were converted into a percentage of cut off thresholds for men and women as described above. Weakness was defined as a result of $<100 \%$ of the cut off threshold while normal strength was considered as being $\geqslant 100 \%$ of this value. To describe and compare the test combinations we calculated the mean of the different populations, the standard error of the mean (SE) and the $95 \%$ confidence interval (CI). Significance was accepted at the level of $95 \%$.

\section{RESULTS}

The most common reason for referral was to investigate neuromuscular diseases and the cause of breathlessness (tables 2 and 3). Data on age, sex and lung function for the three groups are shown in table 4 and the results of the respiratory muscle tests are shown in table 5 .

\section{Global inspiratory muscle tests (Group A)}

One hundred and eighty-two patients completed all inspiratory muscle tests (Pimax, Sniff Pnasal and Sniff Poes, fig 1). Pimax was low in $40.1 \%$, Sniff Pnasal in $41.8 \%$ and Sniff Poes in $37.9 \%$. The correlation coefficient between Pimax and Sniff Pnasal was $r=0.74(p<0.01$, fig 1$)$, between Pimax and Sniff Poes was $r=0.73(p<0.01$, fig 1$)$ and between Sniff Pnasal and Sniff Poes was $r=0.90 \quad(p<0.01$, fig 1$)$. Cross-tabulation (table 6) shows the numbers of patients with low or normal results in all of the tests. Combining the results for the three tests of global inspiratory muscle strength gave a diagnosis of weakness in $29.1 \%$ (table 7 ). This is a relative reduction of 27.4\% compared with PImax alone. Using two non-invasive tests (Pimax and Sniff Pnasal) in combination gave a similar result (low in $32.4 \%$ ).

\section{Diaphragm strength tests (Group B)}

For tests of diaphragm function the 264 clinical referrals who had both Sniff Pdi and Twitch Pdi measurements were analysed (table 5); 68.2\% had weakness when assessed by Sniff Pdi, and $67.4 \%$ when Twitch Pdi was measured. Correlation between Sniff Pdi and Twitch Pdi was $r=0.57$ $(p<0.01$, fig 2$)$. Combining both tests reduced the number of patients considered to have diaphragm weakness to $55.3 \%$ (tables 6 and 8 ), a relative reduction of $18.9 \%$ compared with Sniff Pdi alone.

\section{Expiratory muscle tests (Group C)}

For expiratory muscle strength tests, data from 60 patients who completed measurement of cough Pgas, Twitch T10 and Pemax were analysed; $38.3 \%$ of the patients had expiratory muscle weakness when assessed by Pemax. When assessed by cough Pgas, $36.7 \%$ of the patients had low values and, with Twitch T10, 28.3\% of the patients were considered to be weak. The correlation between Pemax and cough Pgas was $r=0.61$ $(\mathrm{p}<0.01$, fig 3), between Pemax and Twitch T10 $\mathrm{r}=0.28$ $(\mathrm{p}=0.03$, fig 3$)$ and between cough Pgas and Twitch T10 $r=0.63(p<0.01$, fig 3$)$. The combination of all three tests of expiratory muscle strength yielded a diagnosis of weakness in $16.7 \%$ (tables 6 and 9), a relative reduction of 56.4\% compared with Pemax alone.

Table 3 Mean (SD) baseline spirometric parameters of main diagnostic groups

\begin{tabular}{llll}
\hline Groups & FEV $_{\mathbf{1}}(\%$ pred) & VC $(\%$ pred) & FEV $_{\mathbf{1}} /$ FVC $(\%)$ \\
\hline Neuromuscular disease & $67.4(19.0)$ & $65.5(21.1)$ & $74.4(13.3)$ \\
Dyspnoea of unknown cause & $79.2(18.6)$ & $80.1(27.3)$ & $78.4(9.7)$ \\
COPD & $50.9(19.7)$ & $65.7(21.8)$ & $58.8(15.7)$ \\
Rheumatological disease & $62.4(20.5)$ & $65.5(22.0)$ & $78.5(12.9)$ \\
Chest wall disease & $59.8(20.5)$ & $59.8(20.2)$ & $78.8(9.3)$ \\
Other restrictive lung diseases & $51.3(21.4)$ & $54.0(25.2)$ & $76.6(8.1)$ \\
Obesity hypoventilation & $52.3(18.3)$ & $55.7(13.1)$ & $78.2(8.4)$ \\
\hline
\end{tabular}

$\mathrm{FEV}_{1}$, forced expiratory volume in $1 \mathrm{~s}$; VC, vital capacity; FVC, forced vital capacity.

Table 4 Descriptive statistics of the patient subgroups

\begin{tabular}{llll}
\hline & Group A (n= 182) & Group B (n= 264) & Group C ( $\mathbf{n = 6 0 )}$ \\
\hline Sex (M/F) & $109 / 73$ & $160 / 104$ & $44 / 16$ \\
Age (years) & $52.6(14.9)(18-84)$ & $52.3(14.5)(18-85)$ & $51.3(12.3)(24-73)$ \\
$\mathrm{FEV}_{1}(\%$ pred) & $59.4(23.5)(16-115)$ & $61.8(23.5)(16-118)$ & $62.4(25.2)(21-115)$ \\
$\mathrm{FEV}_{1} / \mathrm{FVC}(\%)$ & $73.1(13.1)(33-98)$ & $73.4(12.8)(33-98)$ & $72.9(15.4)(21-95)$ \\
VC (\% pred) & $66.1(23.5)(16-118)$ & $68.0(23.4)(16-143)$ & $68.9(23.1)(23-118)$ \\
$\Delta \mathrm{VC}(\%)(\mathrm{n}=101)$ & $22.1(16.5)(0-71)$ & $20.1(14.2)(0-71)$ & $23.8(14.9)(0-71)$ \\
\hline
\end{tabular}

Values presented as mean (SD) (minimum - maximum).

$\mathrm{FEV}_{1}(\%$ pred), forced expiratory volume in $1 \mathrm{~s}$ as percentage of predicted value; $\mathrm{VC}(\%$ pred), vital capacity as percentage of predicted value; $\triangle \mathrm{VC}$, fall in vital capacity when changing position from sitting to supine. 
Table 5 Respiratory muscle test results for each group

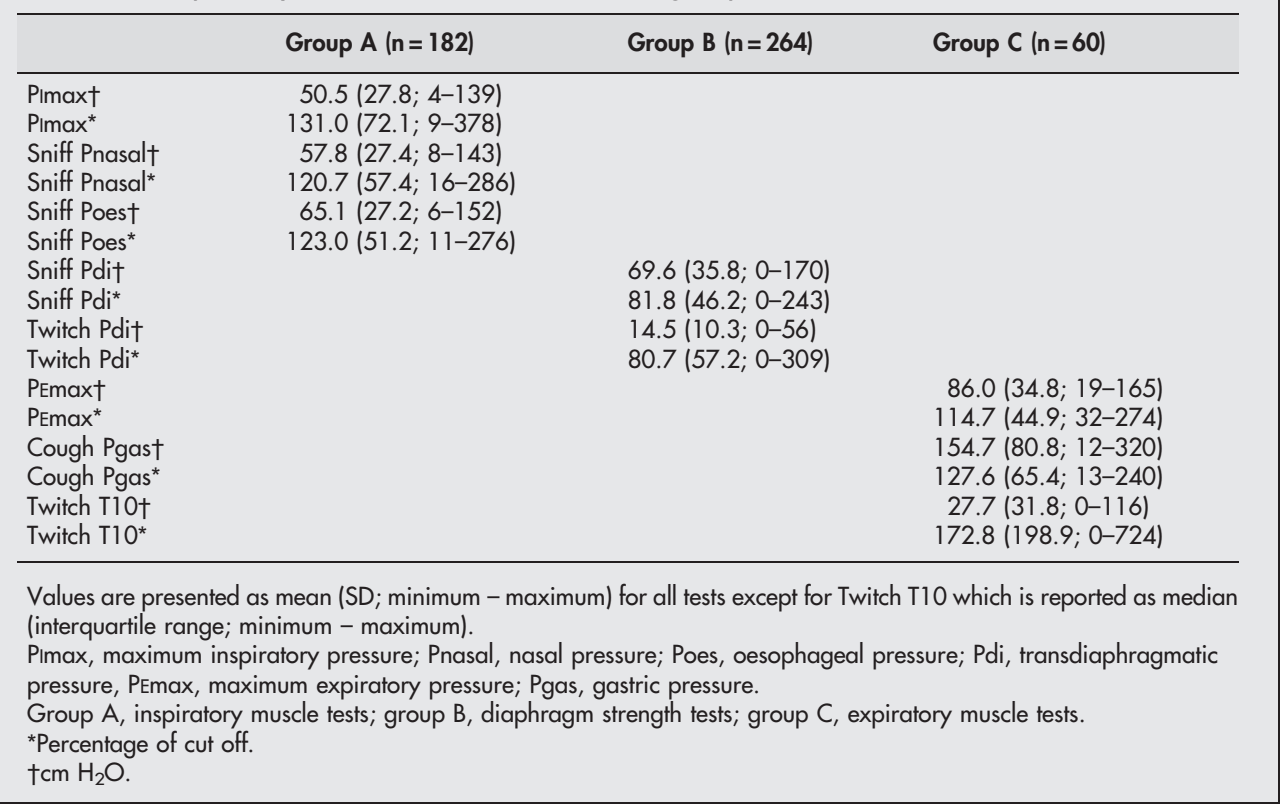
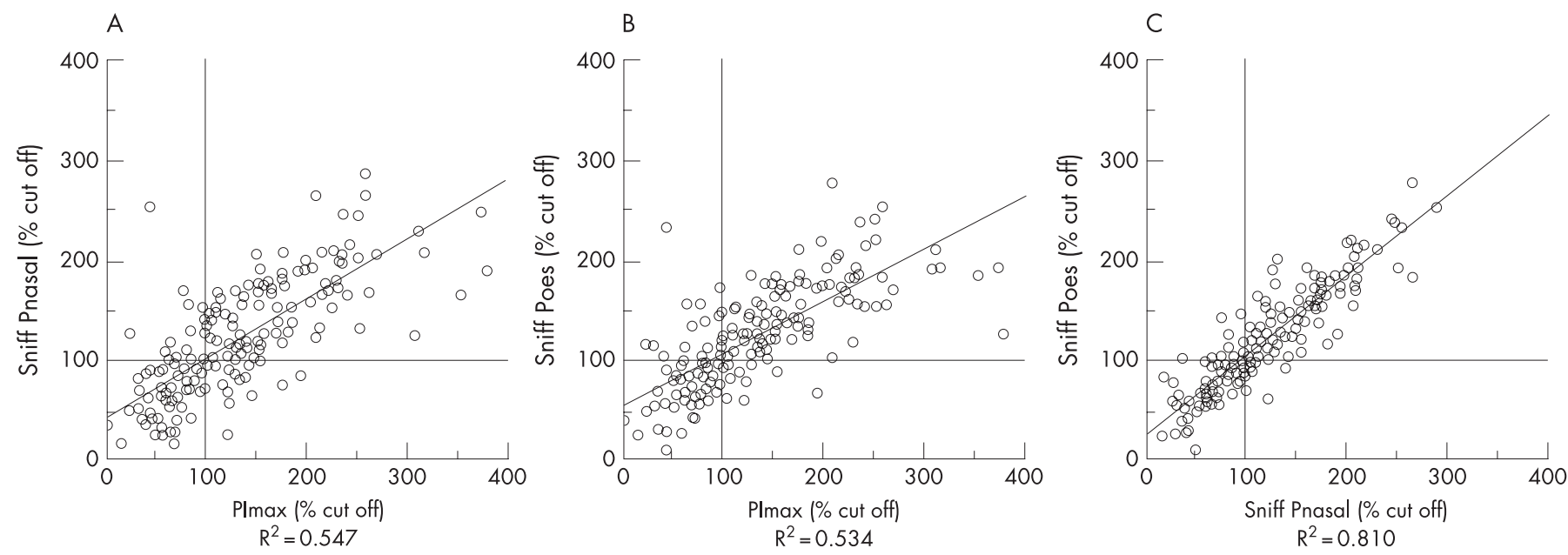

Figure 1 Correlation between (A) maximum inspiratory pressure (PImax) and Sniff nasal pressure (Pnasal), (B) PImax and Sniff oesophageal pressure (Poes) and (C) Sniff Pnasal and Sniff Poes.

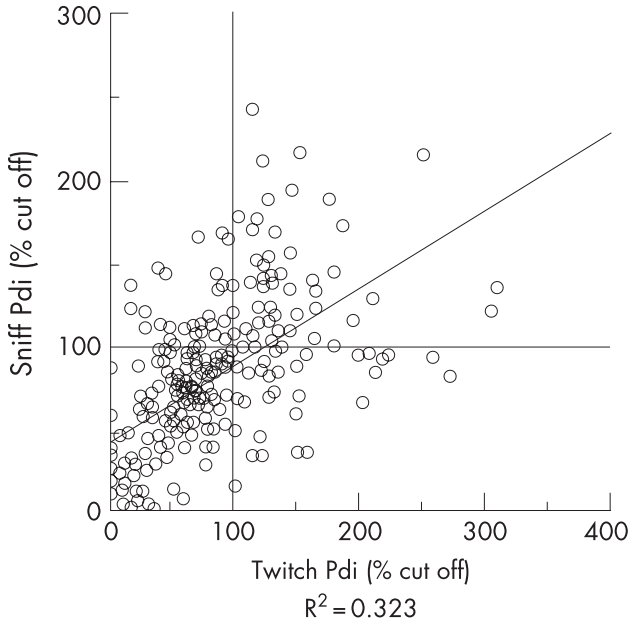

Figure 2 Correlation between Sniff transdiaphragmatic pressure (Pdi) and Twitch Pdi.

\section{DISCUSSION}

PImax and Pemax are widely used, easily applied and noninvasive bedside tests. In our study, Prmax and Pemax diagnosed weakness in $40.1 \%$ and $38.2 \%$, respectively. However, the tests require maximal effort, coordination and cooperation and low values are common and difficult to interpret with confidence. ${ }^{3}$ Sniff Pnasal achieves similar results to PImax, and Sniff Poes-while more precise-is invasive. Compared with Sniff Pnasal, Sniff Poes reduces the diagnosis of weakness by about $10 \%$. The combination of the two non-invasive tests (PImax and Sniff Pnasal) reduces the diagnosis of weakness by about $20 \%$ compared with either test alone. It is of interest that, by performing all three tests, the increase in diagnostic precision is around 30\% compared with PImax or Sniff Pnasal alone, but they are not significantly better than the combination of PImax and Sniff Pnasal. Thus, for patients who are able to sniff and in whom there is likely to be good transmission of intrathoracic pressures (no nasal obstruction or airways obstruction), the combination of the 
Table 6 Cross-tabulation of each test measuring global inspiratory, diaphragm and expiratory strength

\begin{tabular}{|c|c|c|c|}
\hline & \multicolumn{2}{|l|}{ Sniff Pnasal } & \multirow[b]{2}{*}{ Total } \\
\hline & Normal & Low & \\
\hline \multicolumn{4}{|c|}{ Group A: Inspiratory muscle tests } \\
\hline \multicolumn{4}{|l|}{ PImax } \\
\hline Normal & 92 & 17 & 109 \\
\hline Low & 14 & 59 & 73 \\
\hline \multirow[t]{2}{*}{ Total } & 106 & 76 & 182 \\
\hline & $\begin{array}{l}\text { Sniff Poes } \\
\text { Normal }\end{array}$ & Low & Total \\
\hline \multicolumn{4}{|l|}{ PImax } \\
\hline Normal & 94 & 15 & 109 \\
\hline Low & 19 & 54 & 73 \\
\hline \multirow[t]{2}{*}{ Total } & 113 & 69 & 182 \\
\hline & $\begin{array}{l}\text { Sniff Poes } \\
\text { Normal }\end{array}$ & Low & Total \\
\hline \multicolumn{4}{|l|}{ Sniff Pnasal } \\
\hline Normal & 101 & 5 & 106 \\
\hline Low & 12 & 64 & 76 \\
\hline Total & 113 & 69 & 182 \\
\hline \multicolumn{4}{|c|}{ Group B: Diaphragm tests } \\
\hline & Twitch Pdi & & \\
\hline \multicolumn{4}{|l|}{ Sniff Pdi } \\
\hline Normal & 52 & 32 & 84 \\
\hline Low & 34 & 146 & 180 \\
\hline Total & 86 & 178 & 264 \\
\hline \multicolumn{4}{|c|}{$\begin{array}{l}\text { Group C: Expiratory } \\
\text { muscle tests }\end{array}$} \\
\hline & Pemax & & \\
\hline & Normal & Low & Total \\
\hline \multicolumn{4}{|c|}{ Cough Pgas } \\
\hline Normal & 31 & 7 & 38 \\
\hline Low & 6 & 16 & 22 \\
\hline \multirow[t]{3}{*}{ Total } & 37 & 23 & 60 \\
\hline & Twitch T10 & & \\
\hline & \multicolumn{3}{|c|}{ PEmax } \\
\hline Normal & 30 & 7 & 37 \\
\hline Low & 13 & 10 & 23 \\
\hline \multirow[t]{3}{*}{ Total } & 43 & 17 & 60 \\
\hline & Twitch T10 & & \\
\hline & Normal & Low & Total \\
\hline \multicolumn{4}{|l|}{ Cough Pgas } \\
\hline Normal & 36 & 2 & 38 \\
\hline Low & 7 & 15 & 22 \\
\hline Total & 43 & 17 & 60 \\
\hline
\end{tabular}

PImax, maximum inspiratory pressure; Pnasal, nasal pressure; Poes, oesophageal pressure; Pdi, transdiaphragmatic pressure; PEmax, maximum expiratory pressure; Pgas, gastric pressure.

non-invasive tests PImax and Sniff Pnasal is almost as precise as when the invasive Sniff Poes test is added to the assessment.

In this study cough Pgas and Pemax resulted in similar diagnostic outcomes, but the combination of these two volitional tests reduced the diagnosis of expiratory muscle weakness by around 30\% compared with Pemax alone. The combination of all three expiratory tests reduced the diagnosis of weakness by approximately $55 \%$ and was the only combination that reached statistical significance in comparison with the single tests Pemax and cough Pgas.

For the diaphragm specific tests, $68.2 \%$ of the referrals were weak when assessed by Sniff Pdi and $67.4 \%$ by Twitch Pdi. Tests of diaphragm function are complex and relatively invasive. For patients who are able to perform maximum sniff efforts, the Sniff Pdi test is as precise as the Twitch Pdi test and less costly. However, there will be clinical situations in which Twitch Pdi is more appropriate, such as when assessing patients in intensive
Table 7 Combination of PImax, Sniff Pnasal and Sniff Poes results

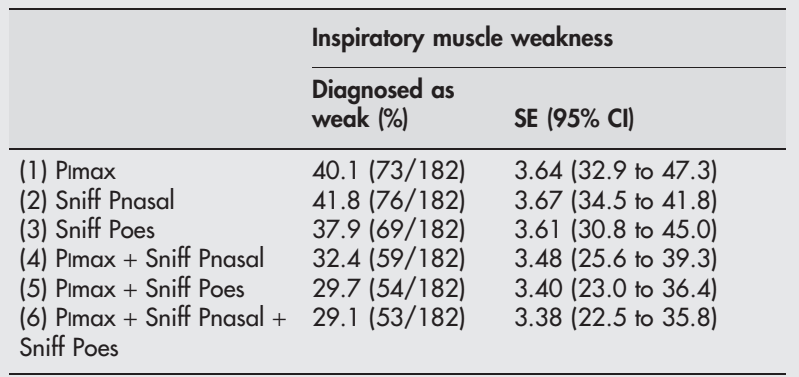

PImax, maximum inspiratory pressure; Pnasal, nasal pressure; Poes, oesophageal pressure; Pdi, transdiaphragmatic pressure, PEmax, maximum expiratory pressure; Pgas, gastric pressure; $\mathrm{SE}$, standard error of the mean; $\mathrm{Cl}$, confidence interval.

Combination of PImax, Sniff Pnasal and Sniff Poes results reduces diagnosis of global inspiratory weakness to $29.1 \%$. This is a relative reduction of 27.4\% compared with PImax alone, 30.4\% reduction compared with Sniff Pnasal alone and $23.2 \%$ reduction compared with Sniff Poes alone. A combination of two tests (4 and 5) reduced the rate to $32.4 \%$ and $29.7 \%$, respectively. There was no significant difference between the single tests. Performing all three tests achieved the highest precision.

care, and the Twitch technique also allows the separate evaluation of each hemidiaphragm. Furthermore, the combination of Sniff Pdi and Twitch Pdi is more precise then either test alone, reducing the relative risk of a false diagnosis of weakness by almost $20 \%$.

The validity of cut off values is important. Tests of respiratory muscle strength can either show normal or low results. A low test result means that the patient is weak as judged by this single test. The different cut off points for each test were taken from the appropriate literature. We compared the published data most appropriate to the methods used at King's College Hospital and Royal Brompton Hospital. The cut off for a normally distributed population was taken for all tests (except the non-normally distributed Twitch T10) by subtracting 1.96SD from the mean for a normal population. This definition is widely accepted for creating cut off values and defining "abnormality". We adopted a similar approach for all tests except Twitch T10 for which the only data available are our own laboratory values. The number of normal subjects for each test reported in the literature is substantial and reproducibility is well described, although we acknowledge that future studies of Twitch T10 will be useful to supplement our own results from 65 normal subjects.

One limitation of this study is a lack of sufficient normal data on the non-volitional tests used to assess diaphragm strength (Twitch Pdi) and expiratory muscle strength (Twitch T10), which does not allow a distinction between reference values for different sexes. More data are needed for Twitch Pdi and Twitch T10 to establish normal values for men and women. Combining male and female data inevitably reduces the sensitivity of the

Table 8 Combination of Sniff Pdi and Twitch Pdi

\begin{tabular}{lll}
\hline & \multicolumn{2}{l}{ Diaphragm weakness } \\
\cline { 2 - 3 } & $\begin{array}{l}\text { Diagnosed as } \\
\text { weak (\%) }\end{array}$ & SE $(95 \% \mathrm{Cl})$ \\
\hline (1) Sniff Pdi & $68.2(180 / 264)$ & $2.87(62.5$ to 73.8$)$ \\
(2) Twitch Pdi & $67.4(178 / 264)$ & $2.89(61.7$ to 73.1$)$ \\
(3) Sniff Pdi + Twitch Pdi & $55.3(146 / 264)$ & $3.07(49.3$ to 61.3$)$ \\
\hline
\end{tabular}

$\mathrm{SE}$, standard error of the mean; $\mathrm{Cl}$, confidence interval; Pdi, transdiaphragmatic pressure.

The combination of Sniff Pdi and Twitch Pdi diagnosed 55.3\% of patients as having diaphragm weakness. The two tests combined reduced the diagnosis of diaphragm weakness by $18.9 \%$ compared with Sniff Pdi alone and 18.0\% compared with Twitch Pdi alone. 

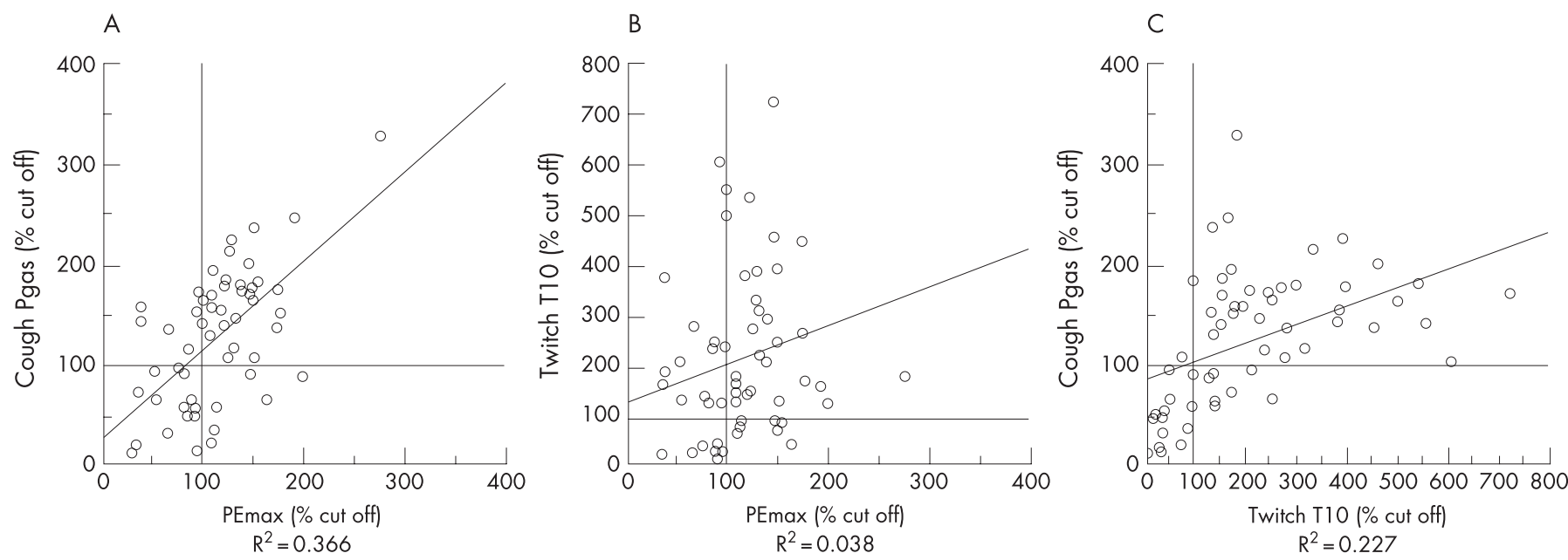

Figure 3 Correlations between (A) maximum expiratory pressure (PEmax) and cough gastric pressure (Pgas), (B) PEmax and Twitch T10 and (C) cough Pgas and Twitch T10.

Table 9 Combination of PEmax, cough Pgas and Twitch $\mathrm{T} 10$

\begin{tabular}{lll}
\hline & \multicolumn{2}{l}{ Expiratory muscle weakness } \\
\cline { 2 - 3 } & $\begin{array}{l}\text { Diagnosed as } \\
\text { weak (\%) }\end{array}$ & SE $(95 \% \mathrm{CI})$ \\
\hline (1) PEmax & $38.3(23 / 60)$ & $6.33(25.7$ to 51.0$)$ \\
(2) Cough Pgas & $36.7(22 / 60)$ & $6.27(24.1$ to 49.2$)$ \\
(3) Twitch T10 & $28.3(17 / 60)$ & $5.87(16.6$ to 40.1$)$ \\
(4) PEmax + cough Pgas & $26.7(16 / 60)$ & $5.76(15.1$ to 38.2$)$ \\
(5) PEmax + cough Pgas + & $16.7(10 / 60)$ & $4.85(8.0$ to 26.4$)$ \\
Twitch T10 & & \\
\hline
\end{tabular}

PEmax, maximum expiratory pressure; Pgas, gastric pressure; $\mathrm{SE}$, standard error of the mean; $\mathrm{Cl}$, confidence interval.

The combination of PEmax, cough Pgas and Twitch T10 diagnosed $16.7 \%$ of the patients to have expiratory muscle weakness and reduced the number of falsely positive diagnoses compared with PEmax and cough Pgas. The relative reduction in false diagnoses was $56.4 \%$ compared with PEmax and $54.5 \%$ compared with cough Pgas.

tests for diagnosing weakness. The relative paucity of Twitch T10 data reduces— but does not negate- - the considerable value of Twitch Tl0 as an expiratory muscle test.

Combining Twitch T10 results with other voluntary tests is helpful as some patients are less good at voluntary tests, but this will reduce sensitivity because the Twitch T10 test has inherent variability, including that due to sex. Despite the fact that the lack of sex-specific data for Twitch T10 reduces the sensitivity of the test, it is noteworthy that the test diagnosed weakness in a slightly higher percentage of cases than the combination of Pemax and cough Pgas.

In summary, the outcome of any one test of inspiratory, specific diaphragm or expiratory muscle strength is broadly similar to any other test. However, a combination of tests can substantially increase the precision of the diagnosis. In many patients it is the assessment of inspiratory muscle strength that is most clinically relevant and the good diagnostic performance of the non-invasive combination of PImax and Sniff Pnasal is important.

\section{ACKNOWLEDGEMENTS}

The authors thank Dr Kazem Rahimi for his help with the manuscript.

\section{Authors' affiliations}

Joerg Steier, Sunny Kaul, John Seymour, Caroline Jolley, Gerrard

Rafferty, William Man, John Moxham, King's College London School of Medicine, King's College Hospital, London, UK

Yuan M Luo, Guangzhou Medical College, Guangzhou Institute of Respiratory Diseases, Guangzhou, China

Michael Roughton, Michael I Polkey, Royal Brompton Hospital, London, UK

JS is the recipient of a long-term research fellowship of the European Respiratory Society (No 18).

Competing interests: None.

\section{REFERENCES}

1 Moxham J. Respiratory muscles. In: Hughes JMB, Pride NB, eds. Lung function tests: physiological principles and clinical applications. London: W B Saunders, 2001:58-68.

2 Moxham J. Respiratory muscle fatigue-aspects of detection and treatment. Bull Eur Physiopathol Respir 1984;20:437-44.

3 ATS/ERS. Joint statement on respiratory muscle testing. Am J Respir Crit Care Med 2002; 166:518-624.

4 Heritier F, Rahm F, Pasche $P$, et al. Sniff nasal inspiratory pressure. A noninvasive assessment of inspiratory muscle strength. Am J Respir Crit Care Med 1994; 150:1678-83.

5 Laroche CM, Mier AK, Moxham J, et al. The value of sniff esophageal pressures in the assessment of global inspiratory muscle strength. Am Rev Respir Dis 1988; 138:598-603.

6 Luo YM, Hart N, Mustfa N, et al. Reproducibility of twitch and sniff transdiaphragmatic pressures. Respir Physiol Neurobiol 2002;132:301-6.

7 Man WDC, Kyroussis D, Fleming TA, et al. Cough gastric pressure and maximum expiratory mouth pressure in humans. Am J Respir Crit Care Med 2003;168:714-7.

8 Miller JM, Moxham J, Green M. The maximal sniff in the assessment of diaphragm function in man. Clin Sci (Lond) 1985;69:91-6.

9 Wilson SH, Cooke NT, Edwards RH, et al. Predicted normal values for maximal respiratory pressures in Caucasian adults and children. Thorax 1984;39:535-8.

10 Leech JA, Ghezzo H, Stevens D, et al. Respiratory pressures and function in young adults. Am Rev Respir Dis 1983;128:17-23.

11 Vincken W, Ghezzo H, Cosio MG. Maximal static respiratory pressures in adults: normal values and their relationship to determinants of respiratory function. Bull Eur Physiopathol Respir 1987;23:435-9.

12 Koulouris N, Mulvey DA, Laroche CM, et al. Comparison of two different mouthpieces for the measurement of Plmax and PEmax in normal and weak subjects. Eur Respir J 1988;1:863-7.

13 Baydur A, Behrakis PK, Zin WA, et al. A simple method for assessing the validity of the esophageal balloon technique. Am Rev Respir Dis 1982;126:788-91.

14 Luo YM, Harris ML, Lyall RA, et al. Assessment of diaphragm paralysis with oesophageal electromyography and unilateral magnetic phrenic nerve stimulation. Eur Respir J 2000;15:596-9.

15 Polkey MI, Duguet A, Luo Y, et al. Anterior magnetic phrenic nerve stimulation: laboratory and clinical evaluation. Intensive Care Med 2000;26:1065-75.

16 Kyroussis D, Mills GH, Polkey MI, et al. Abdominal muscle fatigue after maximal ventilation in humans. J Appl Physiol 1996;81:1477-83. 